\title{
On Analytical Continuation in the Determinantal Method.
}

M. Benayoun and P. Leruste

Laboratoire de Physique Atomique et Moléculaire - Paris

(Lettere al Nuovo Cimento, 1, 548 (1969))

In all the formulae of Sect. $2 a$ ), except relations (1) and (2), please read $P G$ instead of $G$. 